Research Article

\title{
Weighted-Attribute Triplet Hashing for Large-Scale Similar Judicial Case Matching
}

\author{
Jiamin Li $\left(\mathbb{D},{ }^{1}\right.$ Xingbo Liu, ${ }^{1}$ Xiushan Nie, ${ }^{2}$ Lele Ma, ${ }^{1}$ Peng Li, ${ }^{3}$ Kai Zhang, ${ }^{3}$ and Yilong Yin $\left(\mathbb{D}{ }^{1}\right.$ \\ ${ }^{1}$ School of Software, Shandong University, Jinan, China \\ ${ }^{2}$ School of Computer Science and Technology, Shandong Jianzhu University, Jinan, China \\ ${ }^{3}$ Shandong Liju Robot Technology Co., Ltd, Yantai, China \\ Correspondence should be addressed to Yilong Yin; ylyin@sdu.edu.cn
}

Received 22 December 2020; Revised 4 March 2021; Accepted 22 March 2021; Published 16 April 2021

Academic Editor: António Dourado

Copyright (C) 2021 Jiamin Li et al. This is an open access article distributed under the Creative Commons Attribution License, which permits unrestricted use, distribution, and reproduction in any medium, provided the original work is properly cited.

Similar judicial case matching aims to enable an accurate selection of a judicial document that is most similar to the target document from multiple candidates. The core of similar judicial case matching is to calculate the similarity between two fact case documents. Owing to similar judicial case matching techniques, legal professionals can promptly find and judge similar cases in a candidate set. These techniques can also benefit the development of judicial systems. However, the document of judicial cases not only is long in length but also has a certain degree of structural complexity. Meanwhile, a variety of judicial cases are also increasing rapidly; thus, it is difficult to find the document most similar to the target document in a large corpus. In this study, we present a novel similar judicial case matching model, which obtains the weight of judicial feature attributes based on hash learning and realizes fast similar matching by using a binary code. The proposed model extracts the judicial feature attributes vector using the bidirectional encoder representations from transformers (BERT) model and subsequently obtains the weighted judicial feature attributes through learning the hash function. We further impose triplet constraints to ensure that the similarity of judicial case data is well preserved when projected into the Hamming space. Comprehensive experimental results on public datasets show that the proposed method is superior in the task of similar judicial case matching and is suitable for large-scale similar judicial case matching.

\section{Introduction}

Owing to the rapid development of the social economy, the number of judicial cases handled by courts shows a trend of steady increase yearly, and people in the judicial field will endure substantial pressure at work. Among the cases handled by the courts, most were similar. In actual work, handling both common and rare cases requires considerable manpower and material resources. Luckily, algorithms such as machine learning and natural language processing have entered the stage of rapid development and are being broadly applied in our daily life scenarios. It is imperative to integrate artificial intelligence technology into case analysis and trial processes. An important subtask in smart justice is the task of similar judicial case matching. Similar judicial case matching tasks can provide the most similar cases when legal professionals are handling cases, to realize consistent judgement results of similar cases and promote the process of judicial fairness and justice.

Similar judicial case matching is essentially used to determine the similarity of documents. There are previous studies that focused on determining the similarity among documents of different cases. Raghav et al. [1] transformed the document into embeddings using the vector space model and measured the similarity between two embeddings. $\mathrm{Wu}$ et al. [2] used Word2Vec to train the word vectors of legal documents to form the document vector space model and found the most similar top $N$ legal documents from the text matrix. Mueller et al. [3] proposed a twin-network model based on LSTM for calculating semantic similarity. However, when the scale of the judicial case document data to be matched is large, the computational cost of traversing the 
document database will also be large; therefore, the abovementioned method is not suitable for large-scale similar judicial case matching scenarios. In recent years, hashing, a representative method of approximate nearest neighbor search, has garnered widespread attention owing to its low storage and high computational efficiency.

As the most well-known approximate nearest neighbor search method, hashing can map high-dimensional data to compact binary codes in the Hamming space. It has garnered considerable interest in recent years owing to its great efficiency gains in massive data [4-6]. The distance between binary hash codes is measured using the Hamming distance, which can be quickly solved using XOR operations. Therefore, the hash method has great advantages in terms of storage and efficiency $[7,8]$. However, similar judicial case documents are always clustered together in the feature space, and the hash codes mapped from them have a high collision probability, making it difficult to accurately identify them. Therefore, in response to existing problems, the method proposed in this paper introduces the triple loss function to reduce the intraclass distance and increase the interclass distance, making the finally learned hash code more discriminative and improving the accuracy of matching similar judicial documents. At the same time, the hashing method can greatly increase the retrieval speed owing to its binary representation and has great value in improving the efficiency of large-scale judicial case data matching [9].

Similar judicial case matching mainly compares the text similarity of the two judicial documents and selects the most similar document from the two candidate documents, which is essentially the text similarity task. However, owing to the general description framework and the professional words in judicial documents [10], higher requirements are presented for the matching method of similar judicial cases. In comparison with the common text similarity computing task, a similar judicial case matching task faces more challenges. First, judicial case documents are written in a fixed format containing a large number of legal terms, and there are several common words in the factual description part; thus, the mechanism through which to preprocess the judicial document is very important. Second, the length of the judicial documents is quite long, and it is difficult for machines to interpret a long description of facts. Furthermore, the semantic representation is also very complicated. Third, the differences between case documents can be subtle, making it difficult to determine whether the two documents are similar. It can be seen that the feature attributes in the judicial case documents become the key to determining the difference between similar cases. However, efficiently obtaining the appropriate weight of each attribute in judicial case documents remains an open issue.

To overcome the aforementioned limitations, we propose a novel triple deep hashing method, namely, weighted-attribute triplet hashing (WATH), to facilitate similar judicial case matching. Considering the structural complexity of judicial cases document, the proposed method obtains the weight of judicial feature attributes based on hash learning. The binary code of each document is then used to match similar judicial cases in a large corpus.
Our method can benefit the similar judicial case matching in the judicial domain to help the people in the legal field work better and promote the process of judicial fairness and justice. The contributions of this study can be summarized as follows:

This study proposes a similar judicial case matching method based on triple deep hash learning, which performs fast similarity matching by converting judicial case documents into hash codes. Specifically, this method establishes a loss function term based on the similarity of the triplet document, generates the hash code for similarity matching, and improves the matching efficiency.

The weight of each judicial feature attribute is learned using a deep neural network such that the important attributes will get adaptive weights during the hash codes learning procedure.

The experimental results show that the proposed method improves the speed and accuracy of similar judicial case matching and is suitable for large-scale similar judicial case matching.

The remainder of this paper is organized as follows. We briefly introduce related work on similar judicial case matching and representative hashing methods in Section 2. Section 3 presents our proposed approach. Section 4 provides the experimental results on the public dataset. Finally, the conclusions are presented in Section 5.

\section{Related Work}

In this section, we review related works from the following two aspects: similar judicial case matching and hash learning.

2.1. Similar Judicial Case Matching. Similar judicial case matching aims to calculate the similarity between case facts, which is essentially the calculation of text similarity. Similar judicial case matching is a significant practical application of text matching, which is a considerable issue in natural language processing. Currently, there are several models for text similarity calculation in academia. TF-IDF is a statistical method that is often used for text classification and information retrieval. Generally, it only considers the number of documents and the frequency of keywords appearing in documents and requires a lot of training data. The Word2Vec [11] model is a popular text modeling method. It parses legal documents into a collection of words, uses language models to model the contextual relationship between words, and maps words to an abstract low-dimensional real-valued space to generate corresponding word vectors. The bidirectional encoder representations from transformer (BERT) [12] model can be used as a universal language representation model. Its goal is to use a large-scale unlabeled corpus to train and obtain features of text containing rich semantic information.

To date, traditional text matching has been extensively studied, and there are several classic approaches in the field of text matching. Currently, studies on similar judicial case 
matching are mostly conducted on the basis of text similarity tasks, and the method is optimized according to the specific situation of the task. Song et al. [13] used the bus fire incident as an example and established a case similarity matching model by combining the information weight and text similarity calculation methods. Liu et al. [14] established a text similarity model and algorithm for medical dispute cases based on the knowledge of the doctor-patient field. Most studies on text similarity focus on text similarity in general fields, and there are a few text similarity calculation methods in the judicial field. Kumar et al. [15] analyzed the problem of finding similar legal judgements by extending the similarity measure used in information retrieval and web documents. Thenmozhi et al. [16] extracted lexical features from all the legal documents and the similarity between each current case document, and all the prior cases documents are determined using cosine similarity scores. Saravanan et al. [17] compared the extracted features from legal case documents rather than the full texts.

2.2. Hash Learning. Existing learning-based hashing methods can be generally divided into two categories as follows: unsupervised hashing and supervised hashing. Unsupervised hashing methods attempt to construct a hash function on the training data points to preserve the similarity between the original space and Hamming space. Some well-known unsupervised hashing methods include spectral hashing ( $\mathrm{SH})[18,19]$, iterative quantization (ITQ) [20], anchor graph hashing (AGH) [21], discrete graph hashing (DGH) [22], and K-means hashing (KMH) [23]. However, unsupervised hashing methods do not use semantic information. Thus, long codes are required to further improve the search accuracy. Supervised hashing is employed to design the hash function using label information [24, 25]. Representative supervised hashing methods include minimal loss hashing (MLH) [26], supervised hashing with kernels (KSH) [27], fast supervised discrete hashing (FSDH) [28], supervised short-length hashing (SSLH) [29], and supervised discrete hashing with mutual linear regression (SDHMLR) [30].

However, traditional hash functions do not work well for large datasets. Owing to the development of deep learning, deep hashing methods [31-34] achieve higher performance than traditional methods. The design of the loss function is an important part of the deep supervised hashing method. The intuition of the loss function design is to maintain similarity [35]. According to the similarity preserving method, the deep hashing algorithms can be generally divided into pairwise similarity preserving and multiwise similarity preserving. Representative deep hashing methods based on pairwise similarity preserving include deep supervised hashing [36], deep pairwise-supervised hashing [37], deep hashing network [38], and deep asymmetric pairwise hashing [39]. Triplet loss is the most popular loss in the category of deep hashing algorithms which use the multiwise similarity preserving loss function. Representative deep hashing methods based on triplet loss include bitscalable deep hashing [40], one-stage deep hashing [41], and triplet loss hashing [42]. To handle multiview features, some efforts [43-45] and [46] have been made towards effective cross-view hashing.

\section{Proposed Method}

In this section, the proposed WATH method is introduced in detail. We first provide the definition of the problem addressed in this work. Thereafter, we introduce the mechanism through which we can learn the function and obtain the weight of the case feature attributes. Finally, we show the triplet loss function of our model.

3.1. Notation and Problem definition. Without loss of generality, we focus on similar judicial case matching for private lending data. Similar judicial case matching aims to calculate the similarity between case documents. We take a triplet $\left(I, I^{+}, I^{-}\right)_{i=1}^{n}$ as the input, where $I, I^{+}$, and $I^{-}$are fact descriptions of three judicial cases. Case $I$ is the anchor sample, case $I^{+}$is a positive sample, and case $I^{-}$is a negative sample. The aim of hash learning for texts is to learn a mapping $F: I \longrightarrow\{0,1\}^{q}$, such that an input text $I$ can be encoded into $q$-bit binary code $F(I)$. Our goal is to measure case $I^{+}$ and case $I^{-}$with $I$ and predict the case that is more similar to I.

3.2. Model Architecture. In this paper, we propose an architecture of triplet deep hashing designed for judicial similar case matching, as shown in Figure 1. It first represents the characteristics of judicial case documents by BERT. Subsequently, the attributes of the case documents are divided into four parts. Next, the attribute weights are learned to train the hash functions. In addition, judicial case documents are converted into hash codes for fast similarity matching. In this section, we present the details of these parts.

3.2.1. Attribute Feature Extraction Module. Judicial case documents are usually written in a structured format containing many legal terms, and there are several common words in the factual description part; thus, it is hard to select the document most similar to the target document in a large corpus [47]. In addition, the differences between judicial case documents can be subtle, which means that similar judicial case matching is more challenging than normal text matching in information retrieval. It can be seen that the attribute feature in the judicial case documents becomes the key to determine the difference between similar cases. To solve these problems, determining the weight of each attribute of a judicial document becomes fundamental. In comparison with the traditional text-matching model, the method proposed in this paper solves the problem in which different attributes are of equal importance to the matching decision.

Before the documents are sent into the hash function, WATH extracts the judicial feature attributes and converts them into the feature vector using the BERT model. The 


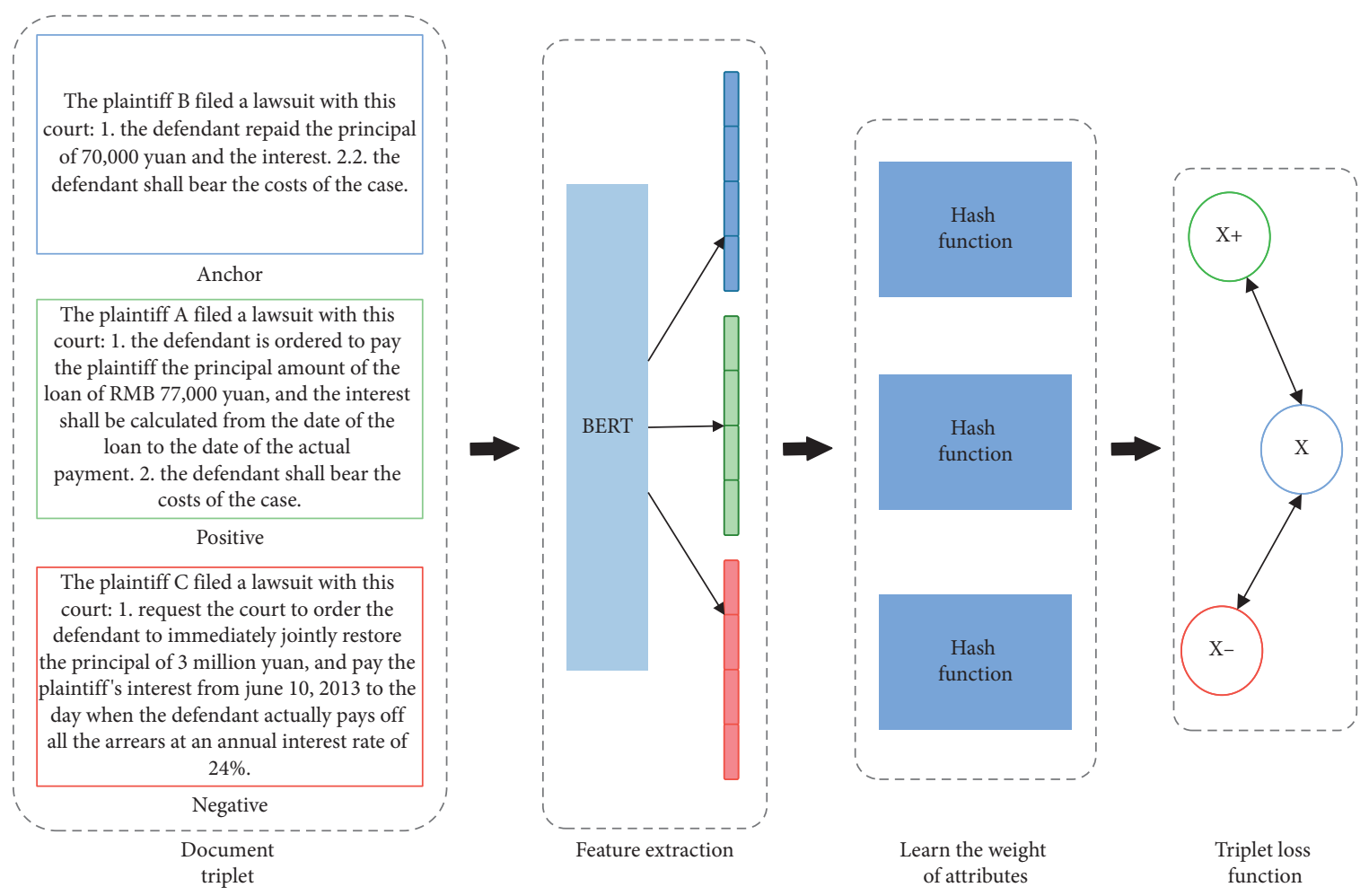

Figure 1: Framework of the proposed approach.

language model BERT [12] is the state-of-the-art model released by Google. It aims to utilize the bidirectional attention mechanism and huge unsupervised corpora to output effective context-sensitive representations of each word in a sentence. In contrast to encoding using term vectors such as Word2Vec and ELMo [48] in the representation layer of the classical natural language model, the BERT model is a pretraining model that can effectively obtain the long-range dependence existing in the document; thus, it can realize encoding of relations between sentences in a similar judicial case text.

We extract a set of specific judicial feature attributes according to the structure and representation of judicial documents. Generally, similar judicial causes will have the same feature attributes in judicial documents. The crucial attributes in private lending for each document include the following: (1) the description of plaintiffs and defendants, including their property and the count of plaintiffs and defendants, (2) the claims of the plaintiff, including the facts and reasons described and the evidence presented by the plaintiff, (3) the defendant's defense, including whether the defendant accepted the fact of the loan and the evidence presented by the defendant, and (4) the statement of the court's decision, including the relationship between the plaintiff and defendant, amount borrowed, and repayment situation.

3.2.2. Learning Hash Function Based on Attribute Weight. After obtaining document attribute feature vectors from the BERT, we propose a deep network module to project these document features into compact hash codes based on weighted attributes. We suppose that each target hash code has $L$ bits. As can be seen in Figure 2, the proposed hash function module first assigns different weights to the input four parts attribute features. Here, the hash function learns the weighted feature attributes of each case document as follows:

$$
\left\{g_{i}\left(x_{i}\right)\right\}_{i=1}^{4},
$$

where $x_{i} \in R^{256}$ are the feature attribute vectors of each document generated by the BERT model and $g_{i}(\cdot)$ is the deep neural network to obtain the weight of different feature attributes.

After the weight of the feature attributes is obtained, we need to combine this weighted feature vector. We use the following two fusion strategies to combine the weighted feature vectors: concatenation and elementwise addition.

(a) Concatenation: the concatenation method directly combines these attributes feature vectors to a superfeature vector so that the input vector can be re-expressed as $g_{1}\left(x_{1}\right)$, $g_{2}\left(x_{2}\right), g_{3}\left(x_{3}\right)$, and $g_{4}\left(x_{4}\right)$. Then, the concatenated vector is

$$
V_{\text {con }}=\left[g_{1}\left(x_{1}\right) ; g_{2}\left(x_{2}\right) ; g_{3}\left(x_{3}\right) ; g_{4}\left(x_{4}\right)\right] \text {. }
$$

We can see that the dimensionality of the concatenation method combined attribute feature vector is $256 \times 4$.

(b) Elementwise addition: another strategy to fuse attribute feature vectors is a parallel combination method. Rather than the concatenation combination method, which combines different attribute feature vectors into a supervector, the parallel combination method combines attribute feature vectors through elementwise addition. The formulas are expressed as follows: 


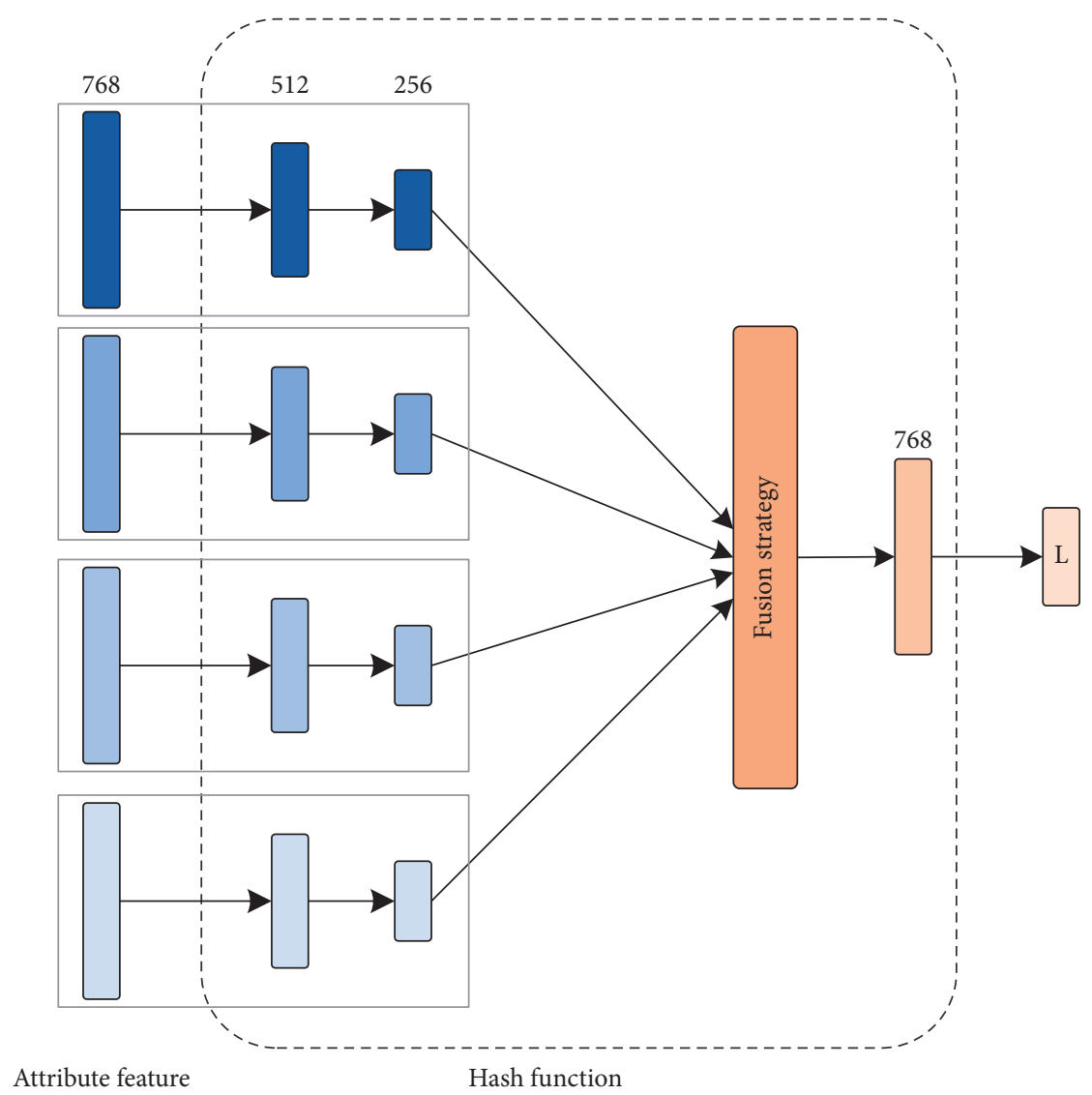

FIgURE 2: Learning hash function based on attribute weight.

$$
V_{\mathrm{add}}=g_{1}\left(x_{1}\right)+g_{2}\left(x_{2}\right)+g_{3}\left(x_{3}\right)+g_{4}\left(x_{4}\right) \text {, }
$$

where + is the elementwise product between two vectors. The dimensionality of the elementwise addition combined attribute feature vector is still 256 . The elementwise addition strategy can enhance the importance of judicial case semantics information.

3.2.3. Loss Function Module. In the existing supervised hashing method, the auxiliary information is mostly in the shape of pairwise labels to indicate that the semantics of the sample pairs are similar or dissimilar. However, a pairwise label only ensures that one constraint is observed. Triplet loss was first proposed in FaceNet by Google to train face embeddings for the recognition task [49]. As demonstrated in Figure 3, triplet loss uses a set of training samples, including an anchor, positive, and negative samples. In particular, triplet loss aims to ensure that the distance between the anchor and positive samples becomes smaller through training and learning, whereas the distance between the anchor and negative samples becomes larger. Therefore, the triplet loss is introduced in this paper.

Specifically, for a given form of instrument sample triplet $\left(I, I^{+}, I^{-}\right)$, the document $I^{+}$is more similar to the query document $I$ than the document $I^{-}$. Such triplet ranking information, i.e., $\operatorname{sim}\left(I, I^{+}\right)>\operatorname{sim}\left(I, I^{-}\right)$is expected to be encoded in the learned binary hash codes. After fusing the four weighted feature attributes, we produce a unified vector representation $V$ of the document. The goal of the method in this study is to learn a mapping $F$ that makes the binary hash code $F(V)$ closer to $F\left(V^{+}\right)$not $F\left(V^{-}\right)$. The loss function based on triples can be expressed as follows:

$$
\begin{aligned}
& L_{\text {triplet }}\left(F(V), F\left(V^{+}\right), F\left(V^{-}\right)\right)=\max \left(0,\left\|F(V)-F\left(V^{+}\right)\right\|_{2}\right. \\
& \left.\quad-\left\|F(V)-F\left(V^{-}\right)\right\|_{2}+\alpha\right), \\
& \text { s.t. } \quad F(V), F\left(V^{+}\right), F\left(V^{-}\right) \in[0,1]^{L},
\end{aligned}
$$

where $\alpha$ is a threshold parameter that measures the distance between matched and unmatched document pairs. In this study, multiple thresholds are used to conduct experiments, and $\alpha$ is set to 0.5 . For a given triplet, this loss function can maximize the distance between the matched document and the unmatched document pair.

3.3. Out-of-Sample Extension. After the network has been trained, we still need to obtain the hashing codes of the case documents that are not in the training data. As shown in Figure 4, for an unseen document triplet, we obtain its binary code by inputting it into the WATH network. Specifically, according to the structure information, an unseen judicial case document is first divided into four parts, and then, we extract the attribute feature as the document 

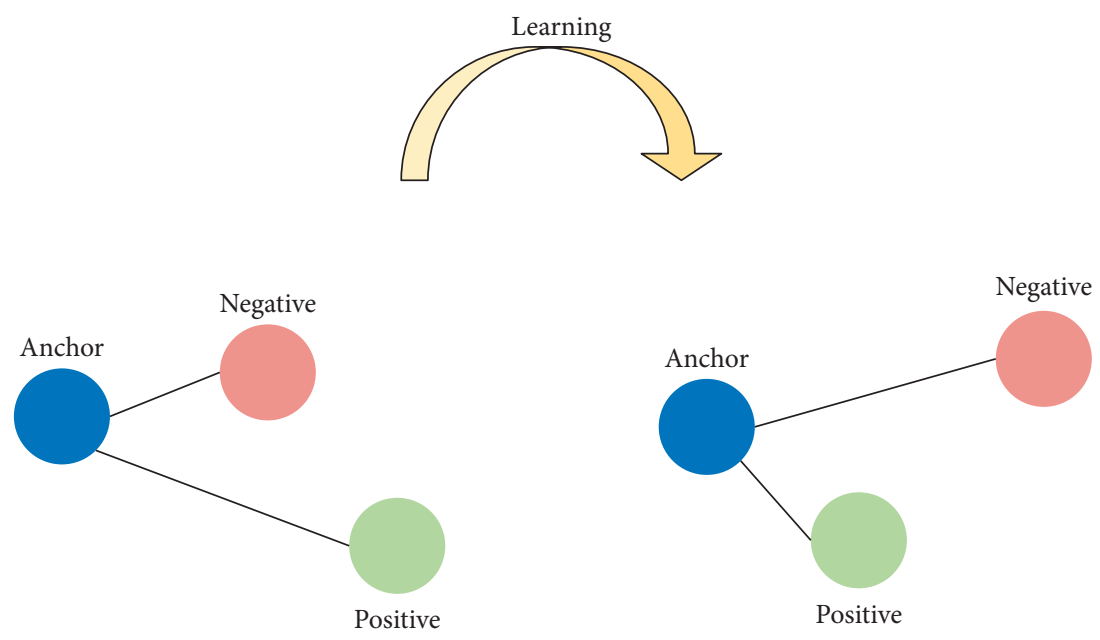

FIGURE 3: Diagram of triple relationship.

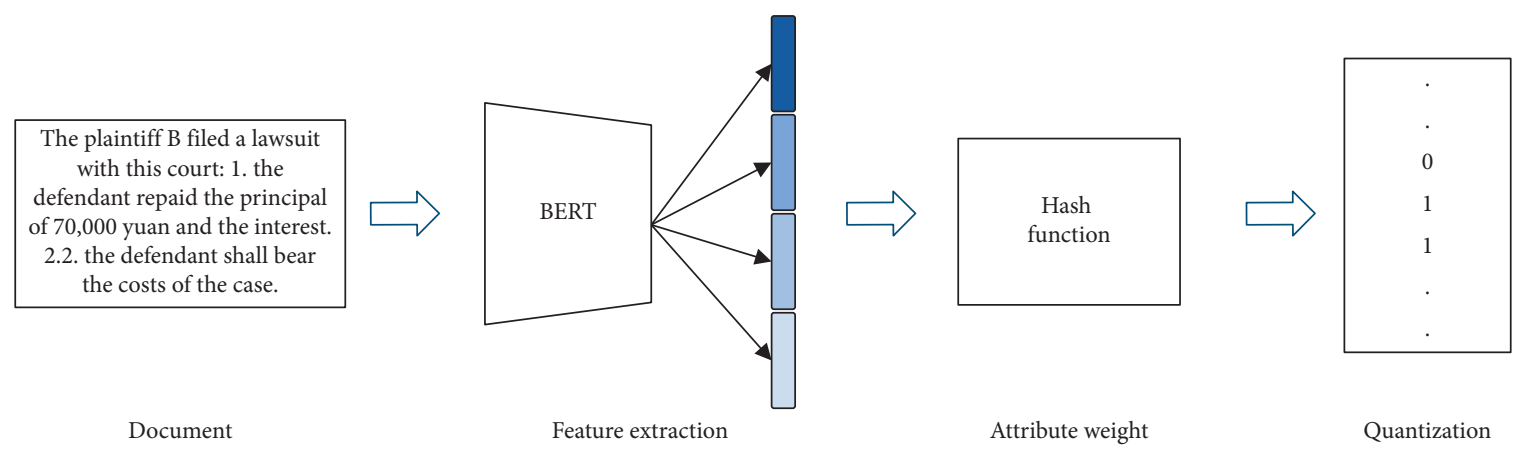

Figure 4: Process of generating hash code for an out-of-sample.

representation using BERT. Subsequently, the weights of the feature attributes are obtained using a hash function. In addition, the attribute feature vector of each judicial case document is fused according to different fusion strategies. Finally, judicial case documents are converted into hash codes for fast similarity matching.

3.4. Computational Complexity. The computational complexity of WATH is composed of three parts: extracting document features, learning binary code, and calculating Hamming distance between documents. The computational complexity of extracting document features is BERT model complexity. The computational complexity of learning binary code is $O\left(n m^{k}\right)$, where $m$ depends on the width of neural networks and $k$ depends on the depth of neural networks. The computational complexity of calculation Hamming distance between documents is $O(1)$.

\section{Experiments}

In this section, we evaluate the match performance of WATH on the public dataset using several representative hashing methods. We first introduce the details of the dataset, evaluation criteria, comparison methods, and implementation details. Thereafter, experimental results and discussions are provided to make fair comparisons. Finally, the convergence of WATH is further investigated.

4.1. Dataset. We conduct experiments on a public dataset from the China Judgements Online website ${ }^{1}$. This dataset contains 5,000 triplets of judicial case documents, which are related to Private Lending. Every document in the triplets contains the fact description part. The documents are presented in triples, whose members are three case documents: one query document $A$ and two documents $B$ and $C$ to be matched. We ensure that the positive sample $B$ is closer to the query document $A$ than the negative sample $C$; that is, $\operatorname{sim}(A, B)>\operatorname{sim}(A, C)$.

4.2. Evaluation Metric. The precision score is used to evaluate the match performance. The precision score measures whether the matched data belong to the same class as the query. The larger the precision score, the better the match performance. The precision score is defined as follows:

$$
\text { Precision }=\frac{\mathrm{TP}+\mathrm{TN}}{\mathrm{ALL}}
$$


TABle 1: Performance in terms of precision scores with deep different lengths of hash code.

\begin{tabular}{lcccccc}
\hline Method & \multicolumn{5}{c}{ Hash bits } \\
& 48 bits & 64 bits & 96 bits & 128 bits & 256 bits & 512 bits \\
\hline SH & 0.4750 & 0.4820 & 0.4850 & 0.4760 & 0.5050 & 0.5120 \\
PCA-ITQ & 0.5066 & 0.5096 & 0.5106 & 0.5196 & 0.5040 & 0.5132 \\
PCA-RR & 0.5114 & 0.5126 & 0.5070 & 0.5186 & 0.5098 & 0.5048 \\
MFH & 0.5244 & 0.5206 & 0.5230 & 0.5258 & 0.5240 & 0.5230 \\
WATH_WS & 0.5790 & 0.5630 & 0.5590 & 0.5800 & 0.5870 & 0.5620 \\
WATH_Con & 0.5988 & 0.5717 & 0.6000 & 0.6030 & 0.5915 & 0.5074 \\
WATH_Add & 0.6060 & 0.5904 & 0.5948 & 0.6018 & 0.5988 & 0.5322 \\
\hline
\end{tabular}

where TP denotes the number of correctly matched data, TN the number of correctly unmatched data, and ALL the total number of samples.

4.3. Baseline Methods. The proposed WATH model is compared with the following five representative hashing methods.

SH [18] first introduces the spectral analysis into the hashing technique, which tries to find the data-dependent compact hash codes such that the neighboring data points in the original Euclidean space are still neighbors in the Hamming space [50].

PCA-ITQ [20] aims to maximize the data variance of each bit via PCA projection and reduces the quantization error between the rotated PCA-embedded data and the discrete codes.

PCA-RR [20] attempts to minimize the quantization error by randomly rotating the datapoints and makes the variance more balanced.

MFH [51] preserves the local structure for each individual feature by considering the local structures of all the features in a global fashion [52].

4.4. Implementation Details. We use the following two different strategies for attribute feature fusion: concatenation and elementwise addition. The concatenation method directly combines these attribute feature vectors to a super feature vector. Another fusion strategy to fuse attribute features is a parallel combination method, which combines attribute feature vectors through elementwise addition.

To further search the role of feature attributes, we designed ablation experiments to evaluate the performance of the method in this study. This is the same as our proposed method, except for the module of weighting feature attributes. The learning rate is set as $10^{-4}$. The batch size and epochs are 128 and 130, respectively. The entire model was optimized using Adam. Specifically, Adam utilizes the first-order momentum $m_{t}$ to retain the direction of the historical gradient. Meanwhile, the second-order momentum $v_{t}$ is used to make the learning rate adaptive for Adam [53]. The Adam update equation can be expressed as follows:

$$
\left\{\begin{aligned}
m_{t} & =\beta_{1} m_{t-1}+\left(1-\beta_{1}\right) g_{t}, \\
v_{t} & =\beta_{2} v_{t-1}+\left(1-\beta_{2}\right) g_{t}^{2}, \\
\widehat{m}_{t} & =\frac{m_{t}}{1-\beta_{1}^{t}}, \widehat{v}_{t}=\frac{v_{t}}{1-\beta_{2}^{t}}, \\
W_{t+1} & =W_{t}-\frac{\eta}{\sqrt{\widehat{v}_{t}}+\varepsilon} \widehat{m}_{t},
\end{aligned}\right.
$$

where $W_{t}$ denotes the $t^{t h}$ iteration. $\beta_{1}$ and $\beta_{2}$ are two hyperparameters that are set to 0.9 and 0.999 , respectively. $\widehat{m}_{t}$ and $\widehat{v}_{t}$ are the bias-current for the first and second moments, respectively. $\epsilon$ is usually set to $1 e-8$, to avoid division by zero.

4.5. Results and Analysis. Table 1 shows the precision scores when we vary the number of hashing bits in $\{64,96,128,256,512,768\}$ on the dataset. It is noteworthy that all the methods generally achieve higher precision scores with an increase in the hash code length. This is reasonable because longer hash codes can encode more semantic information about the document. Nevertheless, the highest precision scores are not always the longest. In particular, we can observe that the proposed WATH approach achieves comparable match performances in different hash length settings and significantly outperforms most baselines, i.e., SH [18], PCA-ITQ [20], PCA-RR [20], and $\mathrm{MFH}$ [51]. The results verify the validity of the attribute weighted and triplet loss. In addition, the Pro_WS method removes the weighted feature attribute vectors, and the accuracy averagely decreases by $2.7 \%$. Thus, we can see that feature attributes play a significant role in the proposed method.

Figure 5 shows the change in the precision score curves with the increase in iterations. The elementwise addition fusion strategy works better than the concatenation fusion strategy because the latter can enhance the importance of judicial case semantics information.

Table 2 shows the results of matching time comparing hash codes with real-valued representation. We investigated the computational time of our proposed methods and compared them with two real-valued methods. The 


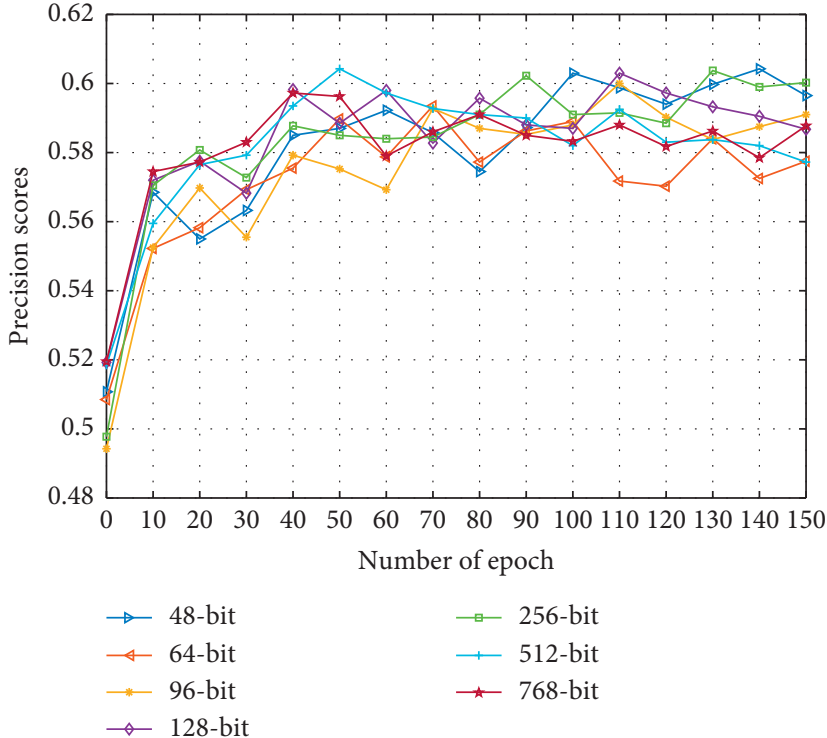

(a)

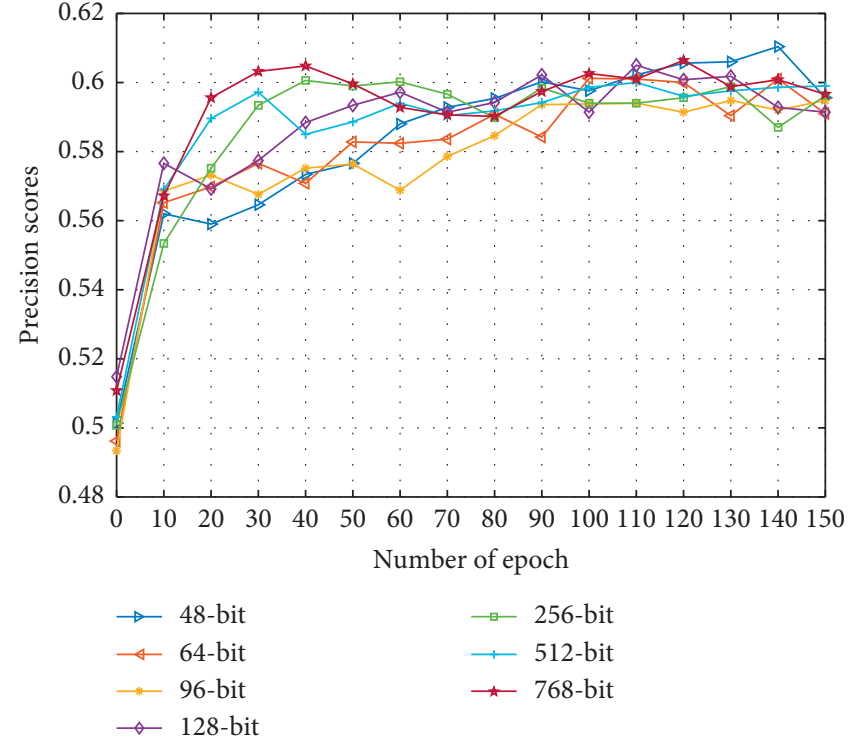

(b)

Figure 5: Precision score with different lengths of hash codes when the number of epochs increases. (a) The results of the utilization of the concatenation fusion strategy. (b) The results of the utilization of the elementwise addition fusion strategy.

TABLE 2: Comparison of matching time (second) using hash codes and real-valued representation.

\begin{tabular}{lcccccccc}
\hline \multirow{2}{*}{ Method } & \multicolumn{4}{c}{ Hash codes } & \multicolumn{3}{c}{ Real value } \\
& 64 bits & 96 bits & 128 bits & 256 bits & 512 bits & 768 bits & Euclidean & Cosine \\
\hline $10^{-5} \mathrm{~s}$ & 0.1698 & 0.1756 & 0.1964 & 0.3016 & 0.4272 & 0.6136 & 35.509 & 77.600 \\
\hline
\end{tabular}
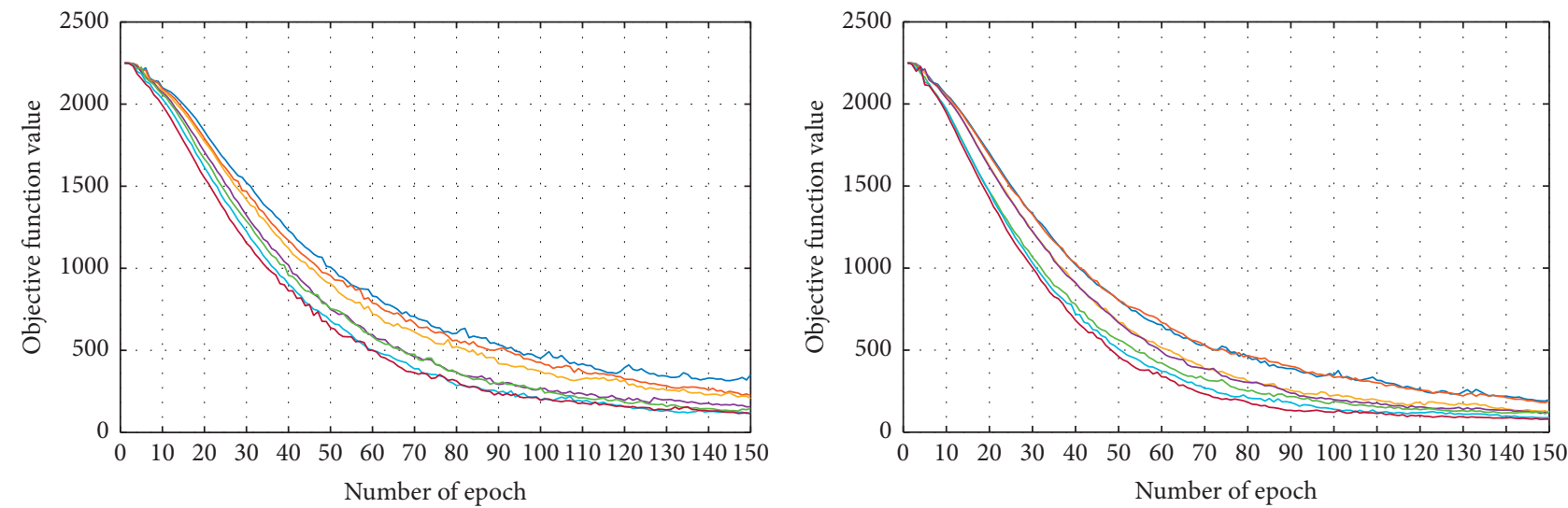

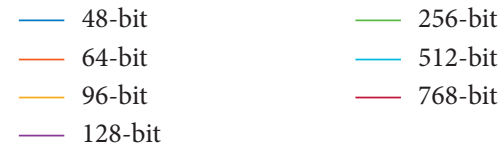

(a)

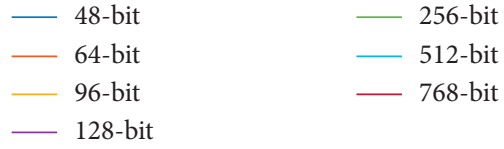

(b)

FIGURE 6: Convergence curves of the proposed method under hash codes with different lengths. (a) The fusion strategy of concatenation. (b) The fusion strategy of elementwise addition. 
matching time corresponds to the time of matching similar case documents in a triplet sample and does not include the feature extraction and the feature attribute vectors weighted. From Table 2, we have the following two observations. First, we can see that the matching time of our proposed hashing method with different hash code lengths is always faster than real-valued representation methods. When we set the code length to 128 bits, the matching speed of the hashing method is approximately 180 and 395 times faster than that of the real value method by using Euclidean distance and Cosine distance, respectively. Second, the storage cost of the realvalued method is 767 times larger than the 128 bit hash code. This is reasonable because each dimension of a binary code can be stored using only 1 bit, whereas several bytes are typically required for one dimension of the real value, leading to a dramatic reduction in storage cost. These results validate the effectiveness of WATH on large-scale datasets.

Figure 6 shows the convergence curves of WATH on the public dataset. We can draw three observations from Figure 6. First, we can observe that the proposed method converges rapidly, usually within 120 iterations of the dataset. The fast convergence speed indicates that the computational costs for learning hash codes are not expensive. Second, it can be observed that the objective function value generally presents a declining trend with the increase in epochs. As the number of epochs increases, the objective function value will eventually remain at a low level for different bits and tends to be stable. Finally, we note that the fitting of our model is better when the size of the bits increases. This implies that the proposed method will more easily distinguish the similarity of samples with an increase in the bit size. These results demonstrate that the proposed WATH has good convergence properties.

\section{Conclusions}

In this paper, we focus on the problem of similar judicial case matching in the judicial case domain and propose the WATH method to solve it. Considering that the judicial case attributes have logical connections, the model divides case documents into four parts according to structure information and then extracts the feature attributes using BERT. One of the main contributions of this paper is that it obtains the weight of different judicial feature attribute vectors by learning a hash function, and we use two fusion strategies to combine the weighted feature attribute vectors. We also introduced triplet constraints to ensure that judicial case data similarity is well preserved when projected into a Hamming space, and case documents are converted into hash codes for fast similarity matching. Comprehensive experimental results on the public dataset and convergence analysis have demonstrated the effectiveness of our algorithm, and the extracted judicial feature attributes are important for large-scale similar judicial case matching. This shows that our proposed method can benefit the similar judicial case matching in the judicial domain to help the people in the legal field work better. In the future, we aim to integrate a variety of loss functions to reduce the reconstruction loss [54].

\section{Data Availability}

Some or all data, models, or code generated or used during the study are available in a repository or online in accordance with funder data retention policies (http://wenshu.court.gov.cn/).

\section{Conflicts of Interest}

The authors declare that they have no conflicts of interest.

\section{Acknowledgments}

This work was supported in part by the National Natural Science Foundation of China (61876098, 61671274, and 61573219), National Key R\&D Program of China (2018YFC0830100 and 2018YFC0830102), and special funds for distinguished professors of Shandong Jianzhu University.

\section{References}

[1] K. Raghav, P. K. Reddy, and V. B. Reddy, Analyzing the Extraction of Relevant Legal Judgments Using Paragraph-Level and Citation Information, p. 30, AI4JCArtifical Intelligence for Justice, 2016.

[2] M. Kamkarhaghighi and M. Makrehchi, "Content tree word embedding for document representation," Expert Systems with Applications, vol. 90, pp. 241-249, 2017.

[3] J. Mueller and A. Thyagarajan, "Siamese recurrent architectures for learning sentence similarity," in Proceedings of the Thirtieth AAAI Conference on Artificial Intelligence, D. Schuurmans and M. P. Wellman, Eds., pp. 2786-2792, Phoenix, AZ, USA, February 2016.

[4] Y. Cao, H. Qi, W. Zhou et al., "Binary hashing for approximate nearest neighbor search on big data: a survey," Institute of Electrical and Electronics Engineers Access, vol. 6, pp. 2039-2054, 2018.

[5] J. Wang, W. Liu, S. Kumar, and S.-F. Chang, "Learning to hash for indexing big data-A survey," Proceedings of the Institute of Electrical and Electronics Engineers, vol. 104, no. 1, pp. 34-57, 2016.

[6] Y. Chen, T. Guan, and C. Wang, "Approximate nearest neighbor search by residual vector quantization," Sensors, vol. 10, no. 12, pp. 11259-11273, 2010.

[7] D. Avola, L. Cinque, A. Fagioli, G. L. Foresti, D. Pannone, and C. Piciarelli, "Bodyprint-A meta-feature based LSTM hashing model for person Re-identification," Sensors, vol. 20, no. 18, p. $5365,2020$.

[8] J. Jung, G. Sohn, K. Bang, A. Wichmann, C. Armenakis, and M. Kada, "Matching aerial images to 3d building models using context-based geometric hashing," Sensors, vol. 16, no. 6, p. 932, 2016.

[9] Y. Qiao, C. Cappelle, Y. Ruichek, and T. Yang, "Convnet and lsh-based visual localization using localized sequence matching," Sensors, vol. 19, no. 11, p. 2439, 2019.

[10] C. Xiao, H. Zhong, Z. Guo et al., "CAIL2019-SCM: a dataset of similar case matching in legal domain, CoRR abs/1911,” 2019, https://arxiv.org/abs/1911.08962, Article ID 08962.

[11] T. Mikolov, I. Sutskever, K. Chen, G. S. Corrado, and J. Dean, "Distributed representations of words and phrases and their compositionality," in Proceedings of the Advances in Neural Information Processing Systems 26: 27th Annual Conference on 
Neural Information Processing Systems 2013, C. J. C. Burges, L. Bottou, Z. Ghahramani, and K. Q. Weinberger, Eds., pp. 3111-3119, Lake Tahoe, NV, USA, December 2013.

[12] J. Devlin, M. Chang, K. Lee, and K. Toutanova, "BERT: Pretraining of Deep Bidirectional Transformers for Language Understanding, CoRR Abs/1810," 2018, https://arxiv.org/abs/ 1810.04805, Article ID 04805.

[13] Y. Song, K. Yu, W. Lyu, and D. Liu, "Research on case similarity matching of city bus fire incidents," International Journal of Innovative Technology and Exploring Engineering, vol. 35, pp. 164-168, 2017.

[14] J. Wang and Y. Dong, "Measurement of text similarity: a survey," Information, vol. 11, no. 9, p. 421, 2020.

[15] S. Kumar, P. K. Reddy, V. B. Reddy, and A. Singh, "Similarity analysis of legal judgments," in Proceedings of the 4th Bangalore Annual Compute Conference, Compute 2011, R. K. Shyamasundar and L. Shastri, Eds., March 2011.

[16] D. Thenmozhi, K. Kannan, and C. Aravindan, "A text similarity approach for precedence retrieval from legal documents," Edited by P. Majumder, M. Mitra, P. Mehta, and J. Sankhavara, Eds., in Proceedings of the Working notes of FIRE 2017-Forum for Information Retrieval Evaluation, vol. 2036, pp. 90-91, Bangalore, India, December 2017.

[17] M. Saravanan, B. Ravindran, and S. Raman, "Improving legal information retrieval using an ontological framework," Artificial Intelligence and Law, vol. 17, no. 2, pp. 101-124, 2009.

[18] Y. Weiss, A. Torralba, and R. Fergus, "Spectral hashing," in Proceedings of the Advances in Neural Information Processing Systems 21, Proceedings of the Twenty-Second Annual Conference on Neural Information Processing Systems, D. Koller, D. Schuurmans, Y. Bengio, and L. Bottou, Eds., Curran Associates, Inc., Vancouver, CA, USA, pp. 1753-1760, December 2008.

[19] Y. Weiss, R. Fergus, and A. Torralba, "Multidimensional spectral hashing," Edited by A. W. Fitzgibbon, S. Lazebnik, P. Perona, Y. Sato, and C. Schmid, Eds., in Proceedings of the Computer Vision-ECCV 2012-12th European Conference on Computer Vision, vol. 7576, pp. 340-353, Florence, Italy, October 2012.

[20] Y. Gong, S. Lazebnik, A. Gordo, and F. Perronnin, "Iterative quantization: a procrustean approach to learning binary codes for large-scale image retrieval," Institute of Electrical and Electronics Engineers Transactions on Pattern Analysis and Machine Intelligence, vol. 35, no. 12, pp. 2916-2929, 2013.

[21] W. Liu, J. Wang, S. Kumar, and S. Chang, "Hashing with graphs," in Proceedings of the 28th International Conference on Machine Learning, ICML 2011, L. Getoor and T. Scheffer, Eds., Omnipress, Bellevue, Washington DC, USA, pp. 1-8, June 2011.

[22] W. Liu, C. Mu, S. Kumar, and S. Chang, "Discrete graph hashing," in Proceedings in the Advances in Neural Information Processing Systems 27: Annual Conference on Neural Information Processing Systems 2014, Z. Ghahramani, M. Welling, C. Cortes, N. D. Lawrence, and K. Q. Weinberger, Eds., pp. 3419-3427, Montreal, Quebec, CA, USA, December 2014.

[23] K. He, F. Wen, and J. Sun, "K-means hashing: an affinitypreserving quantization method for learning binary compact codes," in Proceedings of the 2013 IEEE Conference on Computer Vision and Pattern Recognition, pp. 2938-2945, IEEE Computer Society, Portland, OR, USA, June 2013.

[24] X. Luo, L. Nie, X. He, Y. Wu, Z. Chen, and X. Xu, "Fast scalable supervised hashing," in Proceedings of the The 41st International ACM SIGIR Conference on Research \&
Development in Information Retrieval, SIGIR 2018, K. CollinsThompson, Q. Mei, B. D. Davison, Y. Liu, and E. Yilmaz, Eds., ACM, Ann Arbor, MI, USA, pp. 735-744, July 2018.

[25] Y. Chen, Z. Tian, H. Zhang, J. Wang, and D. Zhang, "Strongly constrained discrete hashing," Institute of Electrical and Electronics Engineers Transactions on Image Processing, vol. 29, pp. 3596-3611, 2020.

[26] M. Norouzi and D. J. Fleet, "Minimal loss hashing for compact binary codes," in Proceedings of the 28th International Conference on Machine Learning, ICML 2011, L. Getoor and T. Scheffer, Eds., Omnipress, Bellevue, Washington DC, USA, pp. 353-360, June 2011.

[27] W. Liu, J. Wang, R. Ji, Y. Jiang, and S. Chang, "Supervised hashing with kernels," in Priceedings of the 2012 IEEE Conference on Computer Vision and Pattern Recognition, pp. 2074-2081, IEEE Computer Society, Providence, RI, USA, June 2012.

[28] J. Gui, T. Liu, Z. Sun, D. Tao, and T. Tan, "Fast supervised discrete hashing," Institute of Electrical and Electronics Engineers Transactions on Pattern Analysis and Machine Intelligence, vol. 40, no. 2, pp. 490-496, 2018.

[29] X. Liu, X. Nie, Q. Zhou, X. Xi, L. Zhu, and Y. Yin, "Supervised short-length hashing," in Proceedings of the Twenty-Eighth International Joint Conference on Artificial Intelligence, IJCAI 2019, S. Kraus, Ed., pp. 3031-3037, Macao, China, August 2019.

[30] X. Liu, X. Nie, Q. Zhou, and Y. Yin, "Supervised discrete hashing with mutual linear regression," in Proceedings of the 27th ACM International Conference on Multimedia, MM 2019, L. Amsaleg, B. Huet, M. A. Larson et al., Eds., ACM, Nice, France, pp. 1561-1568, October 2019.

[31] G. Wu, J. Han, Y. Guo et al., "Unsupervised deep video hashing via balanced code for large-scale video retrieval," Institute of Electrical and Electronics Engineers Transactions on Image Processing, vol. 28, no. 4, pp. 1993-2007, 2019.

[32] G. Wu, Z. Lin, G. Ding, Q. Ni, and J. Han, "On aggregation of unsupervised deep binary descriptor with weak bits," Institute of Electrical and Electronics Engineers Transactions on Image Processing, vol. 29, pp. 9266-9278, 2020.

[33] Q. Li, Z. Sun, R. He, and T. Tan, "Deep supervised discrete hashing," in Proceedings of the Advances in Neural Information Processing Systems 30: Annual Conference on Neural Information Processing Systems 2017, I. Guyon, U. von Luxburg, S. Bengio et al., Eds., pp. 2482-2491, Long Beach, CA, USA, December 2017.

[34] Y. Cao, M. Long, B. Liu, and J. Wang, "Deep cauchy hashing for hamming space retrieval," in Poceedings of the 2018 IEEE Conference on Computer Vision and Pattern Recognition, CVPR 2018, pp. 1229-1237, IEEE Computer Society, Salt Lake City, UT, USA, June 2018.

[35] X. Luo, C. Chen, H. Zhong et al., "A survey on deep hashing methods, CoRR Abs/2003,” 2020, https://arxiv.org/abs/2003. 03369, Article ID 03369.

[36] H. Liu, R. Wang, S. Shan, and X. Chen, "Deep supervised hashing for fast image retrieval," in Proceedings of the 2016 IEEE Conference on Computer Vision and Pattern Recognition, CVPR 2016, pp. 2064-2072, IEEE Computer Society, Las Vegas, NV, USA, June 2016.

[37] W. Li, S. Wang, and W. Kang, "Feature learning based deep supervised hashing with pairwise labels," in Proceedings of the Twenty-Fifth International Joint Conference on Artificial Intelligence, IJCAI 2016, S. Kambhampati, Ed., IJCAI/AAAI Press, New York, NY, USA, pp. 1711-1717, July 2016. 
[38] H. Zhu, M. Long, J. Wang, and Y. Cao, "Deep hashing network for efficient similarity retrieval," in Proceedings of the Thirtieth AAAI Conference on Artificial Intelligence, D. Schuurmans and M. P. Wellman, Eds., AAAI Press, Phoenix, AZ, USA, pp. 2415-2421, February 2016.

[39] F. Shen, X. Gao, L. Liu, Y. Yang, and H. T. Shen, "Deep asymmetric pairwise hashing," in Proceedings of the 2017 ACM on Multimedia Conference, MM 2017, Q. Liu, R. Lienhart, H. Wang et al., Eds., ACM, Mountain View, CA, USA, pp. 1522-1530, October 2017.

[40] R. Zhang, L. Lin, R. Zhang, W. Zuo, and L. Zhang, "Bitscalable deep hashing with regularized similarity learning for image retrieval and person re-identification," Institute of Electrical and Electronics Engineers Transactions on Image Processing, vol. 24, no. 12, pp. 4766-4779, 2015.

[41] H. Lai, Y. Pan, Y. Liu, and S. Yan, "Simultaneous feature learning and hash coding with deep neural networks," in Proceedings of the IEEE Conference on Computer Vision and Pattern Recognition, CVPR 2015, pp. 3270-3278, IEEE Computer Society, Boston, MA, USA, June 2015.

[42] M. Norouzi, D. J. Fleet, and R. Salakhutdinov, "Hamming distance metric learning," in Proceedings of the Advances in Neural Information Processing Systems 25: 26th Annual Conference on Neural Information Processing Systems 2012, P. L. Bartlett, F. C. N. Pereira, C. J. C. Burges, L. Bottou, and K. Q. Weinberger, Eds., pp. 1070-1078, Lake Tahoe, NV, USA, December 2012.

[43] G. Wu, J. Han, Z. Lin, G. Ding, B. Zhang, and Q. Ni, "Joint image-text hashing for fast large-scale cross-media retrieval using self-supervised deep learning," Institute of Electrical and Electronics Engineers Transactions on Industrial Electronics, vol. 66, no. 12, pp. 9868-9877, 2019.

[44] Z. Lin, G. Ding, J. Han, and J. Wang, "Cross-view retrieval via probability-based semantics-preserving hashing," Institute of Electrical and Electronics Engineers Transactions on Cybernetics, vol. 47, no. 12, pp. 4342-4355, 2017.

[45] L. Zhu, X. Lu, Z. Cheng, J. Li, and H. Zhang, "Deep collaborative multi-view hashing for large-scale image search," Institute of Electrical and Electronics Engineers Transactions on Image Processing, vol. 29, pp. 4643-4655, 2020.

[46] L. Zhu, X. Lu, Z. Cheng, J. Li, and H. Zhang, "Flexible multimodal hashing for scalable multimedia retrieval," $A C M$ Transactions on Intelligent Systems and Technology, vol. 11, no. 2, pp. 14-20, 2020.

[47] D. Peng, J. Yang, and J. Lu, "Similar case matching with explicit knowledge-enhanced text representation," Applied Soft Computing, vol. 95, p. 106514, 2020.

[48] M. E. Peters, M. Neumann, M. Iyyer et al., "Deep contextualized word representations," in Proceedings of the 2018 Conference of the North American Chapter of the Association for Computational Linguistics: Human Language Technologies, NAACL-HLT 2018, M. A. Walker, H. Ji, and A. Stent, Eds., vol. 1, pp. 2227-2237, Association for Computational Linguistics, New Orleans, LA, USA, June 2018.

[49] F. Schroff, D. Kalenichenko, J. Philbin, and Facenet, "A unified embedding for face recognition and clustering," in Proceedings of the IEEE Conference on Computer Vision and Pattern Recognition, CVPR 2015, pp. 815-823, IEEE Computer Society, Boston, MA, USA, June 2015.

[50] L. Chen, D. Xu, I. W. Tsang, and X. Li, "Spectral embedded hashing for scalable image retrieval," Institute of Electrical and Electronics Engineers Transactions on Cybernetics, vol. 44, no. 7, pp. 1180-1190, 2014.
[51] J. Song, Y. Yang, Z. Huang, H. T. Shen, and J. Luo, "Effective multiple feature hashing for large-scale near-duplicate video retrieval," Institute of Electrical and Electronics Engineers Transactions on Multimedia, vol. 15, no. 8, pp. 1997-2008, 2013.

[52] D. Hu, F. Nie, and X. Li, "Discrete spectral hashing for efficient similarity retrieval," Institute of Electrical and Electronics Engineers Transactions on Image Processing, vol. 28, no. 3, pp. 1080-1091, 2019.

[53] Y. Zhou, M. Zhang, J. Zhu, R. Zheng, and Q. Wu, "A randomized block-coordinate adam online learning optimization algorithm," Neural Computing and Applications, vol. 32, no. 16, pp. 12671-12684, 2020.

[54] H.-F. Yang, K. Lin, and C.-S. Chen, "Supervised learning of semantics-preserving hashing via deep neural networks for large-scale image search," CoRR Abs/1507, vol. 07, Article ID 00101, 2015. 